\title{
Epidemiological Correlates and Treatment Outcomes among Patients with MDR Tuberculosis in Northern India
}

\author{
Anjana Singh ${ }^{1}$, Ved Prakash', Ravi Kant ${ }^{3}$ \\ All India Institute of Medical Sciences, Rishikesh ${ }^{1}$ \\ King George's Medical University, Lucknow ${ }^{2,3}$.
}

\begin{abstract}
Introduction: Multi drug resistant-TB (MDR-TB) threatens global TB control and is a major public health concern in several countries. The present study was undertaken to detect the epidemiological correlates and treatment outcomes among patients with MDR-TB previously or currently admitted in Department of Respiratory Medicine and Pulmonary and Critical Medicine, KGMU, Lucknow.
\end{abstract}

Material \& Methods: This retrospective study included 2370 TB patients admitted in the Department of Respiratory Medicine and Pulmonary and Critical Medicine, KGMU, Lucknow between years 2012 to 2015. Treatment outcomes were observed. SPSS software was used for data analysis.

Results: The total number of MDR-TB cases enrolled were 2370 . There were $772(32.6 \%)$ males (95\% CI: $30.7 \%-34.5 \%$ ) and 1598 (67.4\%) females (95\% CI: 65.5\% -69.3\%) registered for MDR-TB treatment. The treatment outcomes were as follows: majority $(77.1 \%)$ were under treatment, 279 $(11.8 \%)$ patients were declared cured, $10(0.4 \%)$ were failure cases, while $64(2.7 \%)$ were defaulters, $149(6.3 \%)$ had died and $41(1.7 \%)$ were transferred out.

Conclusion: Emergence of MDR-TB has the potential to be a serious public health problem in Northern India and this necessitates strengthening of TB control and improved continuous monitoring of therapy.

Keywords : MDR-TB, treatment outcome.

\section{Introduction}

Tuberculosis (TB) is a chronic specific bacterial infection caused by Mycobacterium tuberculosis. It primarily affects the lungs (pulmonary tuberculosis) but, in a minority of cases other organs may also be involved (extrapulmonary tuberculosis). India has the largest burden of human tuberculosis in the world, the annual reported cases being 2.2 million. In 2015 , there were an estimated 10.4 million new cases of tuberculosis and 1.8 million deaths due to tuberculosis reported globally (1). Of these, there were an estimated 2.8 million new cases in India, and 0.48 million people died in India due to TB (1). The actual data may not be accurate due to lack of proper identification and reporting (2). Over a million of patients are missing, and government has no data about these missing patients (3). About $80 \%$ of healthcare setup in India is in private sector and as the experience showed, this sector may not report its cases. However, after the legal binding for notifying the TB patient to the government health

Correspondence: Dr. Ved Prakash, Associate Professor, Dept. of Pulmonary and Critical Care, King George's Medical University, Lucknow-226003. 
authorities and penalty clause as mandated by the government of India recently may improve the situation for the better reporting. Thus, until now there is a gross underestimate of the disease due to poor surveillance (2).

Worldwide, anti-tubercular drug resistance is a major public health problem and become hurdle in the TB control programme. Anti-tubercular drug resistance can be with only one drug (mono-resistance) or more than one drugs (poly-resistance). It is called multi-drug resistance-TB (MDR-TB), when mycobacteria is at least resistance to both Rifampicin and Isoniazid. MDR-TB can be primary when infection occurs de novo with the resistant strain or secondary when it is a result of improper drug therapy resulting in to development of resistance to drugs during the course of anti-TB drug therapy. There is scarcity of data on MDR-TB, especially from developing countries. With this background the present study was undertaken to find out the demographic features and outcome of MDR patients enrolled at a tertiary care centre of District Lucknow.

\section{Material and Methods}

This retrospective hospital record based study was conducted in the Department of Respiratory Medicine and Pulmonary and Critical Medicine, KGMU, Lucknow for a period of 4 years from 2012 to 2015. The study protocol was cleared for ethics by research institutional review board. Information on characteristics like age and gender was collected from the patients enrolled under DOTS plus regimen. Treatment outcome were observed.

An MDR-TB case was defined as one whose sputum was culture-positive for Mycobacterium tuberculosis and resistant in vitro to both isoniazid and rifampicin, with or without resistance to other anti-tubercular drugs, on the basis of DST results from an RNTCP certified culture and DST laboratory.
Treatment outcomes were defined as follows:

- Cured: was defined as someone who completed treatment without evidence of treatment failure and who had three or more consecutive negative cultures taken at least 30 days apart, after the completion of the intensive phase.

- Treatment completed: was defined as a patient who had completed treatment but did not meet the definition for cured due to lack of bacteriological results.

- Treatment failure: was defined as treatment terminated or a need for permanent regimen change of at least two anti-TB drugs due to an adverse drug reaction, or lack of culture conversion by the end of the intensive phase, or bacteriological reversion in the continuation phase after conversion to negative after intensive phase, or evidence of acquired additional resistance to fluoroquinolones or secondline injectable drugs.

- Default: was defined as a patient whose treatment was interrupted for two consecutive months or more.

- Death: was defined as those who died for any reason during the course of antituberculor treatment.

\section{Statistical Analysis}

Data entry was made in MS Office Excel software in codes and analysis was done by SPSS software. Descriptive statistical analysis, which included frequency and percentages was used to characterize the data. Association with the factors was tested for significance using Chisquare test and $\mathrm{p}<0.05$ was considered statistically significant. Graphs were made to visualize various findings. 


\section{Results}

The total number of MDR-TB cases enrolled from 2012 to 2015 was 2370 . The number of enrolled cases increased from 61 in the year 2012 to 1027 in 2015 (Fig. 1). There were $772(32.6 \%)$ males (95\% CI: $30.7 \%$ $34.5 \%)$ and $1598(67.4 \%)$ females (95\% CI: $65.5 \%-69.3 \%$ ) registered for anti-TB treatment (Fig. 2). The majority of MDR-TB cases were aged between 21 - 30 years $(37.7 \%)$ followed by those aged less than 20 years $(22.5 \%)$ and those between $31-40$ years $(20.9 \%)$ (Table 1$)$.

\section{Treatment Outcome}

Out of total 2370 patients, majority $(77.1 \%)$ were under treatment; while 279 patients $(11.8 \%)$ were declared cured, $10(0.4 \%)$ were failure cases, while $64(2.7 \%)$ were defaulters, 149 (6.3\%) had died and 41(1.7\%) were transferred out (Table 2). Nearly $57.4 \%$ and 53.4\% cases were cured in 2012 and 2013, respectively while failure rate was 3.3\% in 2012 and $1.8 \%$ in 2013 (Fig. 3). About 1.1\% of patients died and $98.6 \%$ patients were continuing treatment in 2015 (Table 3 ).

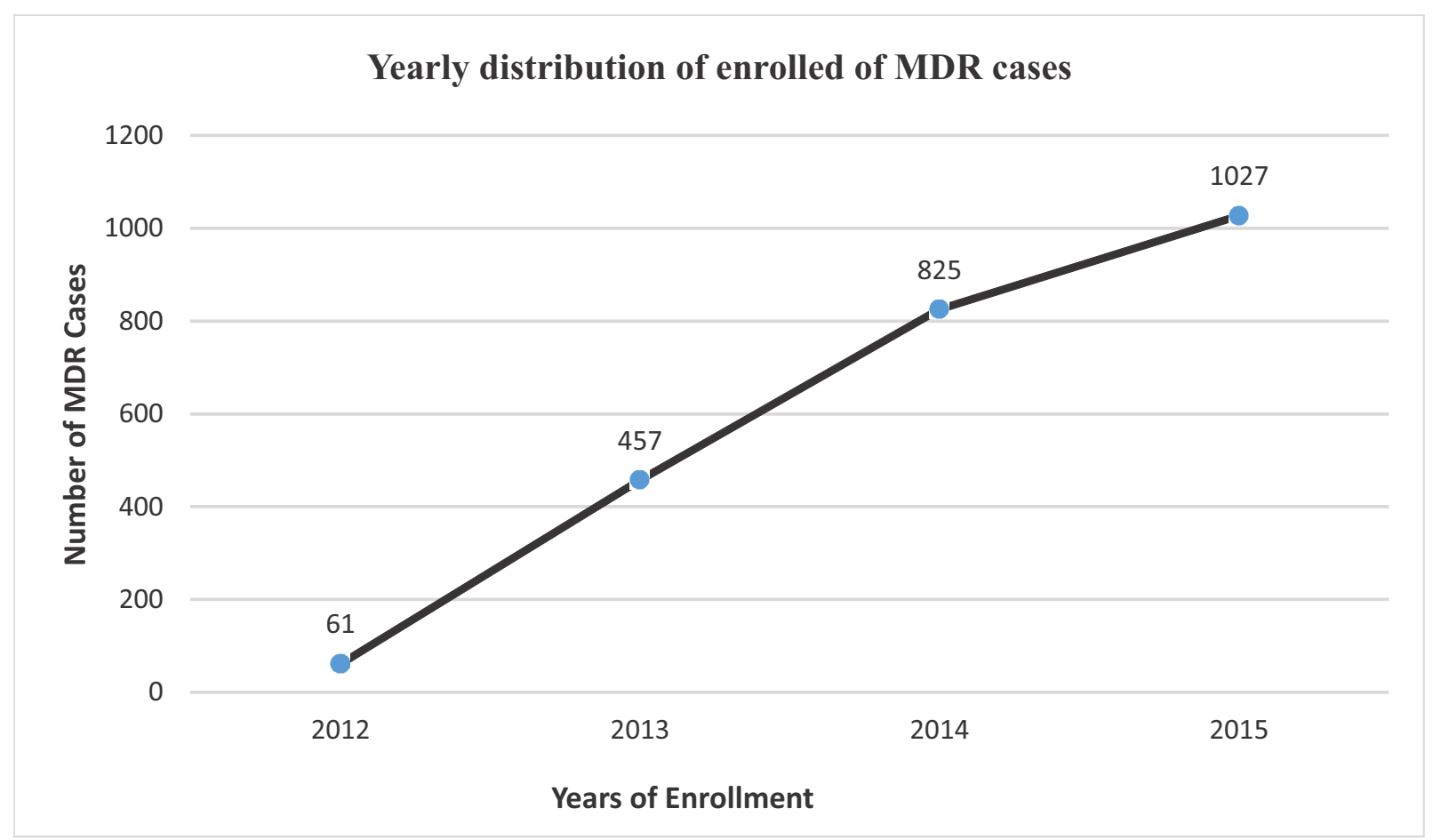

Fig. 1: Yearly distribution of enrolled cases.

Table 1: Age distribution of MDR- TB Cases

\begin{tabular}{|c|c|c|c|}
\hline \multirow{2}{*}{$\begin{array}{c}\text { Age Group } \\
\text { (Years ) }\end{array}$} & \multirow{2}{*}{$\mathbf{N}(\%)$} & Low & 95\% CI \\
\cline { 2 - 4 } & & 20.8 & 24.2 \\
\hline$\leq 20$ & $533(22.5)$ & 35.8 & 39.7 \\
\hline $21-30$ & $894(37.7)$ & 19.3 & 22.6 \\
\hline $31-40$ & $496(20.9)$ & 9.6 & 12.1 \\
\hline $41-50$ & $258(10.9)$ & 4.8 & 6.6 \\
\hline $51-60$ & $135(5.7)$ & 1.7 & 2.9 \\
\hline$\geq 60$ & $54(2.3)$ & & \\
\hline
\end{tabular}


Table 2: Distribution of treatment outcome

\begin{tabular}{|c|c|c|c|}
\hline \multirow{2}{*}{ Treatment Outcome } & \multirow{2}{*}{$\mathbf{N}(\mathbf{\%})$} & Low & 95\% CI \\
\cline { 2 - 4 } & & 10.5 & Upper \\
\hline Cured & $279(11.8)$ & 0.2 & 0.7 \\
\hline Failure & $10(0.4)$ & 2.0 & 3.4 \\
\hline Default & $64(2.7)$ & 5.3 & 7.3 \\
\hline Died & $149(6.3)$ & 75.4 & 2.3 \\
\hline Treatment Continue & $1827(77.1)$ & 1.2 & \\
\hline Transfer out & $41(1.7)$ & & \\
\hline Total & $2370(100)$ & \multicolumn{2}{|}{} \\
\hline
\end{tabular}

Table 3: Year wise distribution of treatment outcome

\begin{tabular}{|c|c|c|c|c|c|c|}
\hline Treatment Outcome & $\begin{array}{c}2012 \\
\mathrm{~N}(\%)\end{array}$ & $\begin{array}{c}2013 \\
\mathrm{~N}(\%)\end{array}$ & $\begin{array}{c}2014 \\
\mathrm{~N}(\%)\end{array}$ & $\begin{array}{c}2015 \\
\mathrm{~N}(\%)\end{array}$ & Chi sq & p-value \\
\hline Cured & $35(57.4)$ & $244(53.4)$ & 0 & 0 & \multirow{7}{*}{2085} & \multirow{7}{*}{$<0.0001$} \\
\hline Failure & $2(3.3)$ & $8(1.8)$ & 0 & 0 & & \\
\hline Default & $6(9.8)$ & 55 (12) & $2(0.2)$ & $1(0.1)$ & & \\
\hline Died & $13(21.3)$ & $108(23.6)$ & $17(2.1)$ & $11(1.1)$ & & \\
\hline Treatment Continuing & $3(4.9)$ & $14(3.1)$ & $797(96.6)$ & $1013(98.6)$ & & \\
\hline Transfer out & $2(3.3)$ & $28(6.1)$ & $9(1.1)$ & $2(0.2)$ & & \\
\hline Total & 61 & 457 & 825 & 1027 & & \\
\hline
\end{tabular}

Gender-wise distribution of enrolled MDR TB cases $(\mathbf{N}=\mathbf{2 3 7 0})$

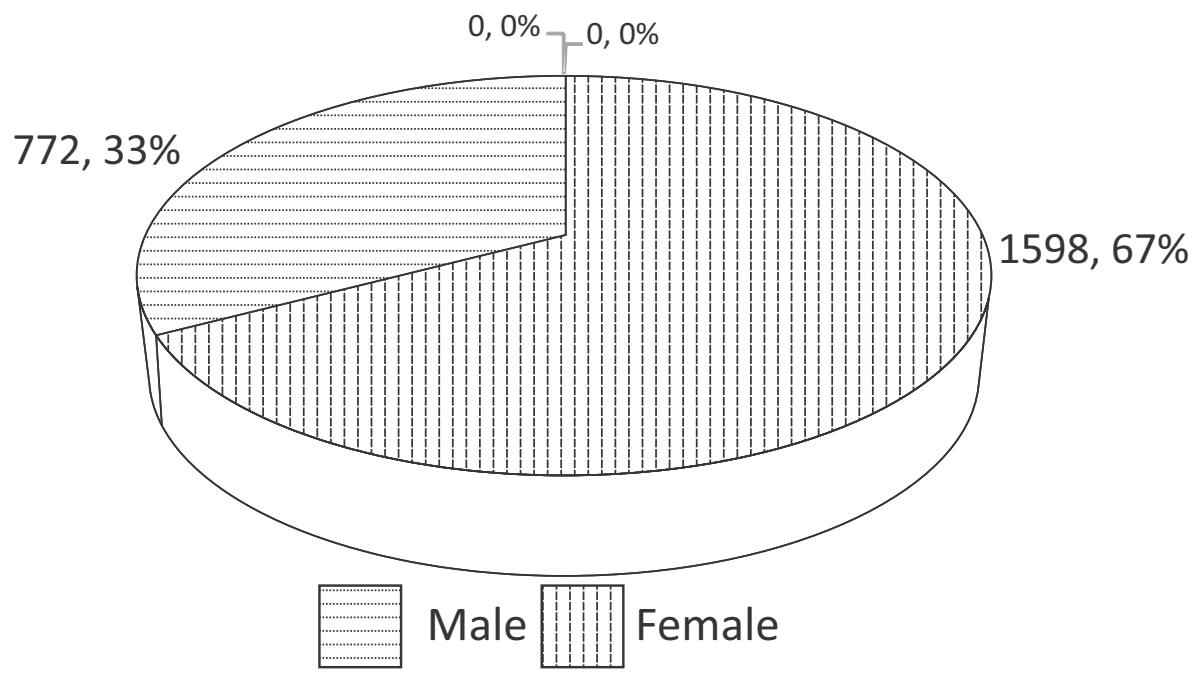

Fig. 2: Gender-wise distribution of MDR-TB cases. 


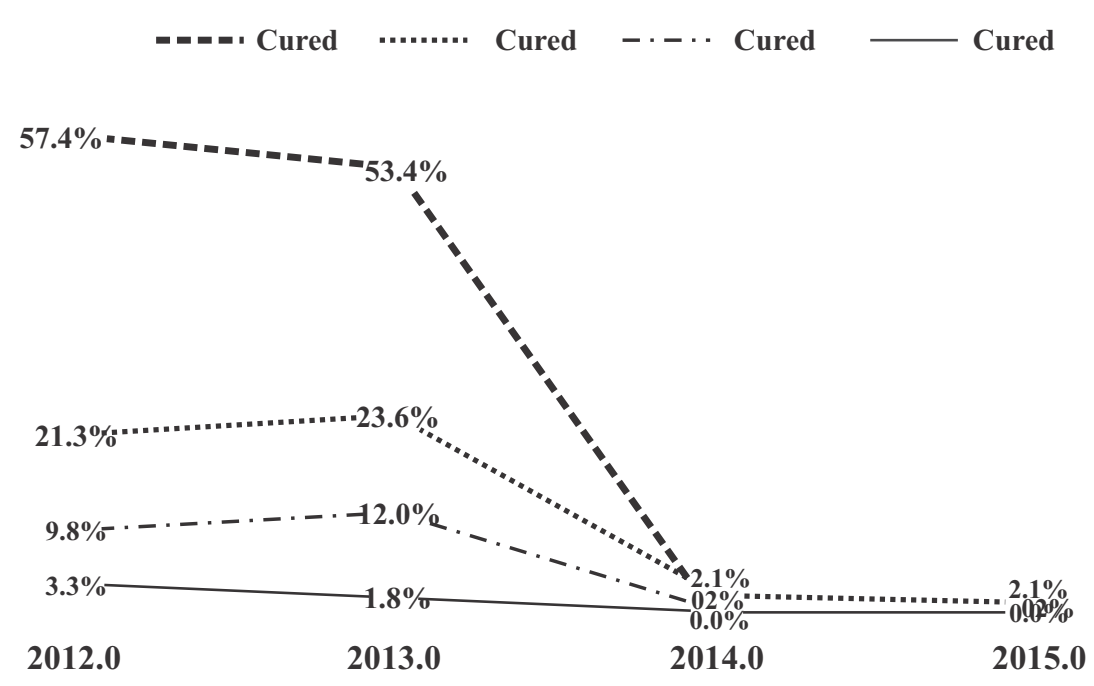

Fig. 3: Year-wise distribution of treatment outcomes.

\section{Discussion}

As per the WHO Global Tuberculosis Report 2018, a total 558,000 (range 483,000 $639,000)$ new cases of MDR-TB estimated in 2017 of which 161,000 new cases were detected and reported. A 139,114 cases (87\%) were started on second line ATT in 2017, up from 129,689 in 2016 (1). The estimated MDR-TB cases in India was 84,000 in 2016 (4). The result of present study shows that the number of MDRTB cases increased from 61 in 2012 to 1027 in 2015. This is again in agreement to the increasing burden of MDR-TB. The greatest burden of TB incidence and mortality in India is in adults aged 15-60 years, which include the most productive members of the society (5). This is similar to the present study results, where TB incidence was higher among those aged up to 50 years. Nearly $57.4 \%$ and $53.4 \%$ cases were cured in 2012 and 2013, respectively. These results are consistent with reported global (52\%) MDR-TB treatment success rates (1). The overall rate of treatment success was $57 \%(95 \%$ CI: $52 \%-61 \%$ ) as reported from a study conducted in China (6). Results from a tertiary referral private hospital in Mumbai with sound mycobacterial laboratory back-up showed 68\% success rates of treatment of a prospective cohort of MDR-TB patients (7). The highest estimated incidence and lower treatment success rates of TB are restricted to countries with low human developmental index (HDI). Life expectancy, education level, gross national income (three essential indicators taken in to accounting the HDI, and urbanisation are significantly associated factors with both TB incidence and treatment success rates (8). As envisaged in sustainable development goals (SDGs), shorter and new MDR-TB regimen needs to be adopted to fulfill the goal of end TB strategy by 2030 (9). In the present study, out of total 2370 patients, majority $(77.1 \%)$ were under treatment, while 279 patients $(11.8 \%)$ were cured, $10(0.4 \%)$ were failure cases while $64(2.7 \%)$ were defaulters, $149(6.3 \%)$ had died and 41(1.7\%) were transferred out. This is comparable to MDR-TB treatment outcome of another study by Ibrahim et al (10) as follows: cured cases were 300 $(52 \%)$, defaulted patients were $54(9.4 \%)$, treatment failure was 17 cases $(2.9 \%), 52$ cases (9\%) completed their treatments, 96 cases $(16.6 \%)$ were still under treatment and 57 cases (9.9\%) died.Adherence to treatment can be attained by strong health education to the patient and their family members prior to start treatment and at different periodic intervals and involvement of community and family in 
providing treatment (11). Primary transmission of MDR-TB can be prevented by improving the social support, living standards, and medical security of each patient (12). Ending TB will not be possible without research. 'India TB Research Consortium (ITRC)' initiative 2016 by the Indian Council of Medical Research (ICMR), is a key step to bring together all major stakeholders to enhance TB research and develop new tools for TB (13).

Long-term follow-up is important for understanding the overall impact of $\mathrm{TB}$ on patient's physical, functional, and socioeconomic well-being. Focus beyond bacteriological cure of MDR-TB patients is needed. Medical management and social support for post-treatment sequelae of MDR-TB should be incorporated in the national programs (14).

\section{References}

1. World Health Organization. WHO/HTM/2018.13. Geneva: World Health Organization; 2018. Global Tuberculosis Report 2018.

2. Chugh TD (2018). Human tuberculosis in India: some neglected issues. Curr Med Res Pract 8(2): 64-66.

3. Subbaraman R, Nathavitharana RR, Satyanarayana S, et al (2016). The tuberculosis cascade of care in India's public sector: a systematic review and meta-analysis. PLoS Med 13: e1002149.

4. Central TB Division, Directorate General of Health Services Ministry of Health and Family Welfare. TB Annual Report 2017. Nirman Bhavan, New Delhi: Central TB Division, Directorate General of Health Services Ministry of Health and Family Welfare; 2014. (Last accessed on 2018 Mar 18).

5. Revised National Tuberculosis Control Programme. Technical and Operational Guidelines for Tuberculosis Control in India 2016.

6. Alene KA, Hengzhong Yi, Viney K, et al (2017). Treatment outcomes of patients with multidrug-resistant and extensively drug resistant tuberculosis in Hunan Province, China. BMC Infect Dis 17: 573.

7. Udwadia ZF, Moharil G (2014). Multidrug-resistant-tuberculosis treatment in the Indian private sector: results from a tertiary referral private hospital in Mumbai. Lung India 31(4): 336-341.

8. Khazaei S, Rezaeian S, Baigi V, et al (2017). Incidence and pattern of tuberculosis treatment success rates in different levels of the human development index: a global perspective. South Afr $J$ Infect Dis 32(3): 100-104.

9. Prasad R, Gupta N, Banka A (2017). Rapid diagnosis and shorter regimen for multidrug-resistant tuberculosis: a priority to improve treatment outcome. Lung India 34:1-2.

10. Ibrahim E, Baess AI, Messery MAA (2017). Pattern of prevalence, risk factors and treatment outcomes among Egyptian patients with multidrug resistant tuberculosis. Egypt J Chest Dis Tuberc 66: 405-411.

11. Moitra S, Sen S, Mukherjee S, Das P, Sinha S, Bose M (2015). Study of prevalence and outcome of standardized treatment on category I pulmonary tuberculosis cases in North India: A single center experience. Community Acquir Infect 2:83-92.

12. Kumar A, Singh AK, Upadhyay V, Pandey J (2018). Epidemiology of multi-drugresistant tuberculosis in Northern India. Biomed Biotechnol Res J2: 112-121.

13. Dias HM, Pai M, Raviglione MC (2018). Ending tuberculosis in India: A political challenge \& an opportunity. Indian J Med Res 147: 217-220.

14. Singla R, Mallick M, Mrigpuri P, Singla N, Gupta A (2018). Sequelae of pulmonary multidrug-resistant tuberculosis at the completion of treatment. Lung India 35: 48. 